\title{
From Paper to Module - An Integrated Environment for Generating SCORM Compliant Moodle Courses Out of Text and Multimedia Elements
}

\author{
Hans-Martin Pohl, Benedikt Deicke, and Jan-Torsten Milde \\ Fulda University of Applied Science \\ HCI Research Center \\ Heinrich-von-Bibra-Platz 3, 36037 Fulda, Germany \\ hans-martin.pohl@verw.hs-fulda.de, benedikt@synatic.net, \\ jan-torsten.mildedinformatik.hs-fulda.de
}

\begin{abstract}
ECampus is a project spanning all departments at the University of Fulda. It has been started to create a uniform learning environment at the university. The objective is to research and develop a user-friendly easy-to-use editor to generate SCORM 2004 conform E-Learning modules. This editor is based on Open Source software and new technologies such as XSL transformations and the Google web toolkit. The whole system is provided as a web application and embedded within the E-Learning environment of the university. Some E-Learning modules can be developed with the system immediately. These modules are now being used during the lessons with great success.
\end{abstract}

Keywords: SCORM 2004, XSLT, transformation, creation of content, E-Learning, modules, lesson, user friendly, style sheet, LOM, moodle, LMS.

\section{Introduction}

For the average author it is almost impossible to generate a SCORM 2004 file structure by hand. SCORM, as a technical standard, combines a number of existing ELearning specifications and formats. A SCORM compliant E-Learning unit may consist of arbitrary files containing the actual content of the unit. In most cases, the unit's textual content is stored in (X)HTML files, which can be displayed with a standard web browser. Multi media files, e.g. images, flash animations, audio and video files, are stored separately in their proprietary formats.

Only a small number of SCORM editors exist (e.g. Reload, http://www.reload.ac.uk/scormplayer.html). Even with these systems, the user still has to have a good understanding of the SCORM file structure. This technical barrier is much too high for most tutors. As a result, standard compliant E-Learning content is not produced. In this paper we describe an integrated environment, that facilitates the simple creation of such content. 


\section{State of the Art}

While there are currently a lot of different Learning Management Systems, the use is limited to but a few in Germany. Hereby Moodle (see http://www.moodle.org) and 'Ilias' (see http://www.ilias.de/ios/) play the greatest role.

In contrast to the LMS there are only a limited number of editors for creating an ELearning module. HTML editors are widely-use but don't provide adequate functionality. Furthermore E-Learning modules generated by an editor can mostly only be used within a proprietary LMS.

Additionally, a lot of effort is required to learn how to use these editors. Such a system fails entirely if there has to be consistent development and presentation of tests and learning success.

The advantage of these systems is the functionality for communication between users or between tutor and student. In our case this can be neglected because this functionality is captured by the LMS 'System2Teach' (see also http://www.system2teach.de) which is a proprietary development of the University of Applied Sciences Fulda. This paper focuses on easy and comfortable development of E-Learning modules based on textual content by using the eCampus framework.

Concerning the technology for state of the art web applications there are many choices. Many different systems support the web based approach. Besides PHP (see http://www.php.net) or JSP (see http://java.sun.com/products/jsp/index.jsp) it is also possible to use Ruby (see http://www.ruby-lang.org) in combination with the Ruby on Rails framework (see http://www.rubyonrails.com).

\section{The eCampus Project}

This research focuses on the development of a user-friendly system for writing E-Learning units, allowing authors to concentrate on the content and the didactic concept of the unit, instead of worrying about the underlying technology.

It is part of the eCampus project at the University of Applied Sciences Fulda. In this interdisciplinary project, content is being created for the faculties Nutritional Sciences, Food and Consumer Sciences, Applied Computer Science, Nursing \& Health Care as well as Food Technology. Within the first years of the project, many E-Learning modules were produced. All modules address prominent introductory courses of the particular program of study. This was only possible by using the eCampus framework which is described below.

The central target of the project is the implementation of a tool that decreases the complexity of the process of learning module production. The central objectives of the learning module production are an increase in the interactivity of the course, as well as an increase in the quality and quantity of self learning.

Currently LMS with SCORM 2004 support are not commonly available. Until Moodle is SCORM 2004 capable the format of transformed E-Learning modules will be SCORM 1.2. 


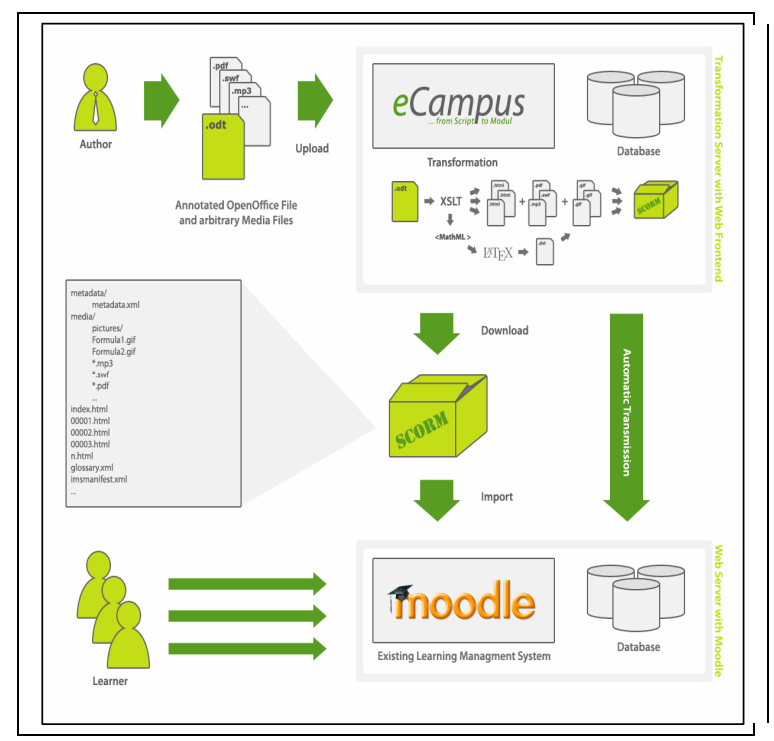

Fig. 1. System Architecture

\subsection{Overview System Architecture}

The learning module production takes place in three phases (see Figure 1):

- the structural annotation of the textual content

- the automatic generation of the E-Learning unit

- the automatic transmission into the LMS and allocation as SCORM compliant E-Learning module

In phase one, most of the (textual) content is created in a standard word processor (e.g. Open Office, [10]). Here the text is structurally annotated using a predefined formatting template. The text is stored and automatically converted into a simple XML file, which provides the basis for subsequent transformation steps. This approach works very well for static content and is used in a number of systems (e.g. eLAIX (see $h t t p: / / w w w . b o l d t-m e d i a . d e /)$ ).

In the second phase, the file prepared in phase one is transformed into a SCORM 2004 compliant E-Learning module automatically. For this, the document is unpacked and analyzed. To get by on cascaded XSL transformations (EXtensible Stylesheet Language) each element is identified and transformed with specific instructions (see Figure 2). Finally all contents are packed into an E-Learning module and multiplexed with the necessary auxiliary files.

The available metadata is separated and attached to the module in a LOM (Learning Objects Metadata) compliant form.

The complete process was formerly controlled by the Apache ant build tool ([4]) but is now ported to Rake (see http://rake.rubyforge.org). It uses saxon8 (see http://saxon.sourceforge.net/) as a transformation engine. 


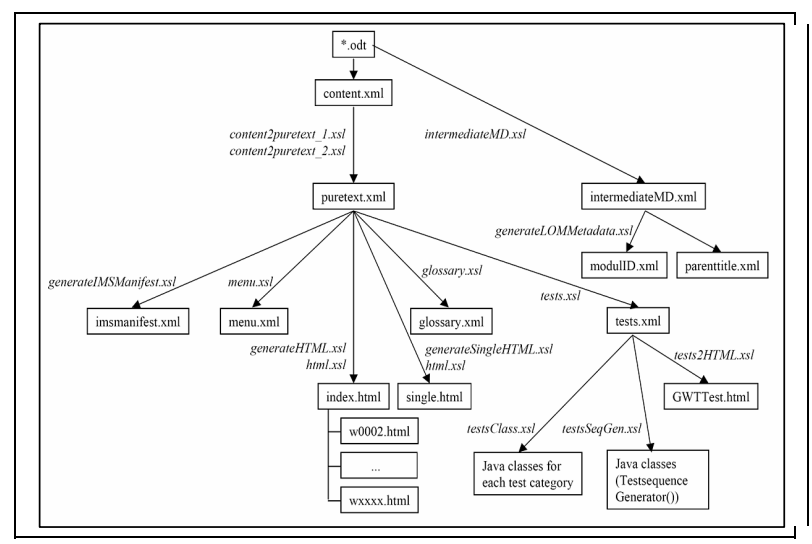

Fig. 2. Transformation Tree of eCampus Framework

In the last phase the author is able to either download the education module or to transfer it to a Moodle server, where a new course is created automatically. Unfortunately Moodle currently lacks a stable web service API. Due to this the automatic transfer and creation of courses is not yet supported until Moodle fully implements this.

In addition the transformation server itself also provides a RESTful Web service API. These interfaces make it possible to integrate the transformation server into other applications.

\subsection{User Front End}

The user front end is very simple and easy to use. All main functions can be controlled by the main screen (see Figure 3 ).

The menu bar on top contains the administration functions and an online help.

The main part is the dark green beam with the input box for the source file. If the path is unknown the file can be selected using the browser's file chooser. Afterwards the transformation can be started with a single click. In advanced mode there is the possibility to set the name of the target file or assign a style for the presentation as single HTML files within a browser. Additionally it is possible to add media files like images, flash animations, audio or video.

The lower part of the screen lists all transformations and displays their current state. It is possible to get a detail view and to download the results of completed transformations. The detail view (see Figure 4) displays the state of the transformation and contains the included source files, the resulting learning units and some functions to reannotate or retransform the source file.

\subsection{Transformation Process}

The complete process is controlled by the Rake (see http://rake.rubyforge.org) build tool and uses saxon8 (see http://saxon.sourceforge.net/) as a transformation engine supported by a graphical user interface (GUI). 


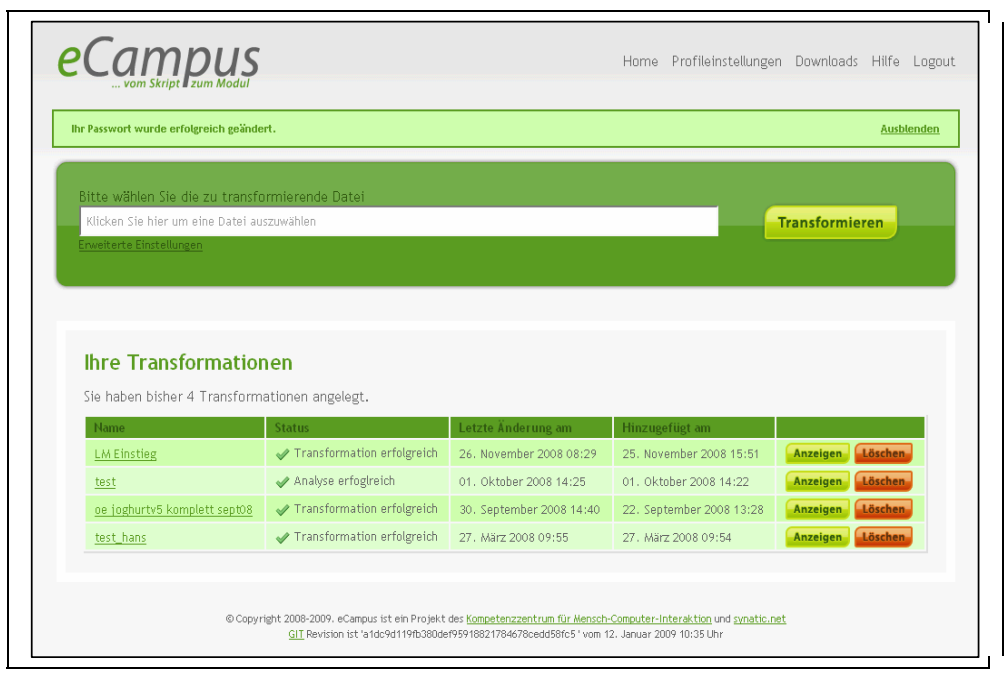

Fig. 3. User Front End Main Screen

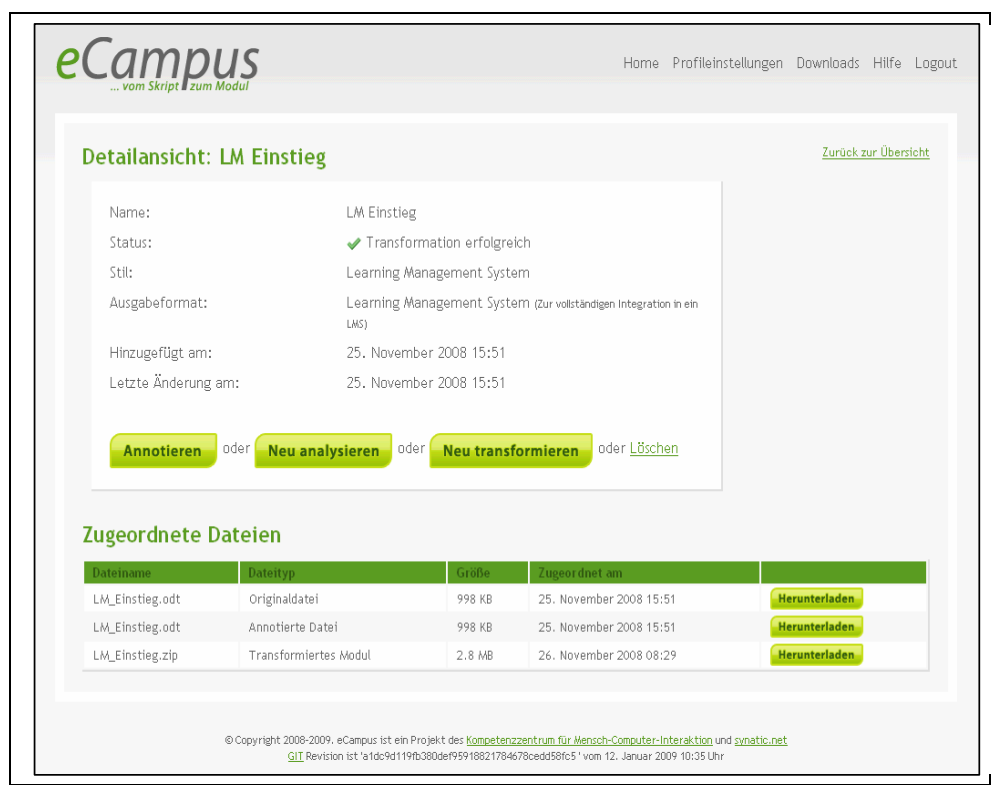

Fig. 4. Detail View of a Transformation

After starting transformation, the written Open Office document is unpacked. After that the file 'content.xml', which contains the content, is accessible and can now be transformed (see Figure 4).

The first step of the transformation is to transform this 'content.xml' into a new file called 'puretext.xml'. This new file is in an intermediate format. Thereby the 
annotated elements are assigned an explicit format template. Furthermore all elements without annotation are deleted. The result of this transformation is a universal intermediate format without direct correlation to the producer application.

The transformation is controlled by the XSLT stylesheets 'content2pure_1.xsl' and 'content2pure.xsl'.

The advantage of this intermediate format is that the resulting transformations are totally independent of the content collecting process or application. As a result a content collecting process using other applications is also possible in the future, e.g. Microsoft Word. Only the transformation into the intermediate format has to be adapted.

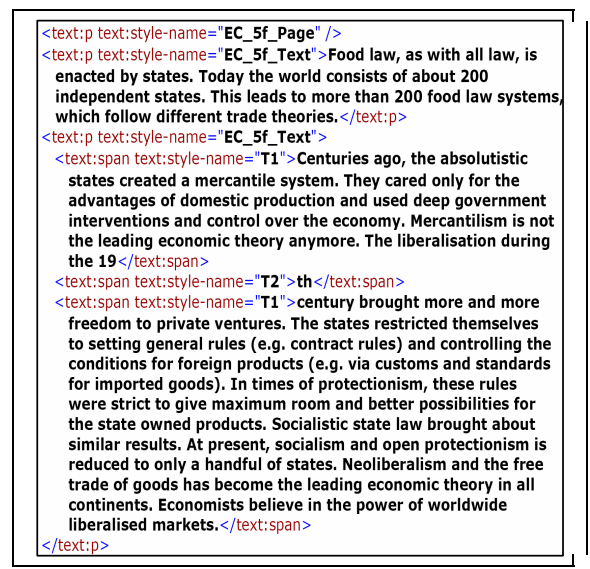

Fig. 5. Abstract of the unpacked *.odt

The file in intermediate format can be transformed into the output format (here HTML) by applying the XSLT stylesheets 'generateHTML.xsl' and 'html.xsl'

For this purpose each element from 'puretext.xml' is analyzed and the corresponding transformation instruction is searched. If the correct instruction is found, it is applied to the specific element. The transformation result is stored into the HTML file.

To print out the content easily a further version is generated. In contrast to the first form all learning objects, sessions and sections, are stored within one page. This page can easily be printed by using the print functionality of the browser.

An overall glossary is generated by applying the stylesheet 'glossary.xsl'. Hereby all elements which are annotated as glossary entries are transformed into a separate glossary file.

To use the learning module without an LMS there must be extra navigation. In this case all headlines from sections and sessions are transformed into the file 'menu.xml' supported by 'menu.xsl'

The metadata which is stored within the 'content.xml' is read and transformed into a further intermediate format 'intermediateMD.xml'. From this the 'generate LOMMetadata.xsl' generates the 'modulID.xml' and 'parenttitle.xml' which 
conforms to the LOM standard. Subsequently the complete content and all metadata is transformed into XML files.

\subsection{Integration in LMS}

It is possible to manually download and import the resulting ZIP archive into any SCORM compliant LMS. Additionally, as the University of Applied Sciences Fulda is currently using Moodle, it is planned to implement an automatic transfer and import of generated learning modules into this LMS. Unfortunately Moodle's SOAP based web service API is currently under development and scheduled for its 2.0 release. As soon as the required functionality is implemented, the eCampus web application will be extended accordingly. By clicking a single button the user will be able to automatically create a new course in the Moodle LMS based on the previously generated learning unit.

\section{Discussion and Prospects}

As the transformation process is run on a single central server, it becomes relatively simple to update and extend the system functionality. An expensive deployment onto multiple computers is not needed anymore and it is ensured that all authors are always able to use the up-to-date transformation process.

The transformation server is based on the dynamic scripting language Ruby and the programming language Java. Both are using virtual machines which are available for various operating systems. Although the transformation server is currently running on Linux it is possible to run it on other platforms, too.

Future works will be concentrating on the development of a web based editor making it possible to replace OpenOffice. This editor should be able to import documents in different formats, automatically analyse the document structure and provide functions to annotate these documents online.

\section{References}

1. Crockford, D.: RFC 4627. The application/json Media Type for JavaScript Object Notation (JSON), http: / / www . j son . org/ (accessed, 13.01.2009)

2. David Eisenberg, J.: OASIS OpenDocument Essentials. LuLu.com (2004)

3. Hodgins, W., Duval, E., et al.: Draft Standard for Learning Object Metadata. Report, Learning Technology Standards Committee. IEEE, Los Alamitos (2002)

4. Holzner, S.: Ant - The Definitive Guide. O’Reilly Media, Sebastopol (2005)

5. Google Inc. GWT: the Google Web Toolkit, http: / / code.google.com/webtoolkit/ (accessed 13.01.2009)

6. Kay, M.: XSLT 2.0 programmer's reference. Wiley, Indianapolis (2004)

7. Koper, R.: Modeling units of study from a pedagogical perspective (2001), http://eml.ou.nl/introduction/articles.html

8. Advanced Distributed Learning. SCORM 2004 (2004), http: //www.adlnet.gov/scorm (accessed, 13.01 2009) 
9. Schulmeister, R.: Grundlagen hypermedialer Lernsysteme: Theorie - Didaktik - Design. Oldenbourg Verlag, München (2002)

10. OpenOffice.org team. OpenOffice, http://www.openoffice.org/ (accessed 13.01.2009)

11. W3C. XML, http: / /www.w3 . org/MarkUp/ (accessed, 13.01.2006)

12. Wilde, E., Lowe, D.: XPath, XLink,XPointer, and XML. Addison-Wesley Professional, Reading (2002) 